\title{
L'APPORT DE LA BIOGÉOGRAPHIE DANS L'ÉTUDE DE LA BIODIVERSITÉ
}

\author{
Dr. Jérôme Fournier*
}

\section{RESUMO:}

A biodiversidade, tema central deste artigo, está atualmente ameaçada. Pesquisar as causas de sua erosão, analisando os mecanismos, prevendo suas consequências, e, em seguida, proteger o que pode ser protegido, sāo um dos maiores desafios aos quais a comunidade cientifica se confronta. A biogeografia e a ecologia são as disciplinas metadisciplinares que melhor integraram a diversidade das pertubaçōes de origem antrópica. O declínio generalizado da biodiversidade se traduz pelo desaparecimento progressivo das populações locais e pela fragmentação dos habitats. Esta erosão reduz as diversidades local (alfa) e regional (gama), empobrece o tecido das comunidades, rompe as interaçōes mútuas e deteriora os ciclos biogeoquimicos. Os geógrafos podem participar neste desafio. Os instrumentos de sensoriamento remoto e os Sistemas de Informação Geográfica (SIG) são de grande utilidade para o estudo diacrônico dos fenômenos em escalas locais, regionais e continentais. A análise da fragmentação dos ecossistemas pode permitir uma melhor compreensão dos mecanismos em jogo e ajudar a estabelecer áreas protegidas. A contribuiçāo dos geógrafos é, portanto, complementar à dos biólogos da conservação e à dos ecologistas.

\section{PALAVRAS-CHAVE:}

Biodiversidade, biogeografia, ecologia, gestāo, meio ambiente.

\section{RÉSUMÉ:}

La biodiversité, thème central de cet article, est aujourd'hui menacée. Rechercher les causes de son érosion, en analyser les mécanismes, prévoir ses conséquences, puis protéger ce qui peut l'être, sont les plus grands défis auxquels la communauté scientifique est confrontée. La biogéographie et l'écologie sont des disciplines métadisciplinaires et celles qui ont le mieux intégré la diversité des perturbations d'origines anthropiques. Le déclin généralisé de la biodiversité se traduit par la disparition progressive des populations locales et la fragmentation des habitats. Cette érosion réduit les diversités locale (alpha) et régionale ( $\mathrm{gamma}$ ), appauvrit le tissus des communautés, rompt les interactions mutualistes et détériore les cycles biogéochimiques. Les géographes peuvent participer à ce défi. Les outils de télédétection et les Systèmes d'Information Géographique (SIG) sont d'une grande utilité pour l'étude diachronique des phénomènes aux échelles locales, régionales et continentales. L'analyse spatiale de la fragmentation des 
écosystèmes peut permettre de mieux comprendre les mécanismes en jeu et aider à la mise en place d'aires protégées. L'approche des géographes est donc complémentaire à celle des biologistes de la conservation et des écologues.

\section{MOTS-CLÉS:}

Biodiversité, biogéographie, écologie, gestion, environnement.

\section{Introduction}

La biodiversité représente la variété de la vie animale et végétale de la biosphère. Elle est devenu le thème prioritaire des discussions internationales relatives à la protection de la nature (CHAUVET \& OLIVIER, 1993; MAC NEELY, 1990; MAGURRAN, 1988). Sa définition figure dans l'article 2 de la Convention sur la diversité biologique: "Biological diversity means the variability among living organisms from all sources including, interalias, terrestrial, marine and other aquatic ecosystems and the ecological complexes which they are parts; this includes diversity within species, between species and of ecosystems"

La biodiversité n'est pas un concept ni un paradigme. Ce mot reste flou car il évoque la pluralité et les relations entre éléments constitutifs de cette pluralité. La biogéographie peut ètre définie comme "l'étude spatio-temporelle des diversités biologiques, de leur origine, de leur évolution et de leur régulation dans des espaces hétérogènes et changeants" (BLONDEL, 1995): elle étudie donc elle aussi d'une certaine manière la biodiversité. Il est possible de définir la biodiversité comme "la quantité et la structure de l'information contenue dans des systèmes vivants hiérarchiquement emboités" (BLONDEL, 1995).

Deux problèmes interdépendants intéressent aujourd'hui les scientifiques: la quantification et le maintien de la diversité biologique. La quantification représente l'estimation en termes de nombres mais aussi et surtout comment ces nombres varient de lieu en lieu. C'est là, un des aspects majeurs de la recher- che en biogéographie. La biodiversité quantitative permet de localiser, géographiquement, les richesses biologiques de notre planète. Connaitre la distribution spatiale des écosystèmes d'une région et le nombre d'espèces associées permet une meilleure gestion du territoire. Ce thème de recherche est l'objet d'une nouvelle méta-discipline en pleine émergence: la biologie de la conservation. Il s'agit là d'un travail que les géographes, et en particulier les biogéographes, peuvent mener à bien en collaboration avec les autres disciplines scientifiques. Dans notre monde, comprendre la biodiversité devient essentiel puisqu'il s'agit de ressources limitées que nous savons fragiles. Cette étude se situe résolument à l'interface entre Nature et Société.

\section{Les niveaux d'analyse de la biodiversité}

Il est possible de hiérarchiser la Nature en niveaux d'intégration biologique: gènes, cellules, organismes, populations, espèces, communautés, écosystèmes, puis paysages et géosystèmes, biomes et biosphères (BARBAULT, 1994). Cette approche s'intéresse aussi aux diversités réalisées à différentes échelle d'espace. On reconnait trois principaux niveaux organisationnels de la diversité biologique, celui des gênes, celui des espèces et celui des écosystèmes. Ce dernier niveau est plus difficile à évaluer que les deux autres car les limites entre les écosystèmes ne sont pas nettes. La diversité écosystémique est essentiellement étudiée au niveau local ou régional et évaluée d'après la diversité d'espèces végétales (phanérogames) et 
animales (oiseaux, insctes) faciles à voir et à déterminer. La variation génétique est le fondement de la biodiversité car ce sont à cause de leurs différences génétiques que les espèces diffèrent entre elles. Le nombre de gènes est évalué, par exemple, à $1000 \mathrm{chez}$ les bactéries, 10000 chez les champignons, à $400000 \mathrm{chez}$ certaines plantes à fleurs. Cette diversité est accrue par le nombre très important d'individus qui existent chez certaines espèces. Les 10000 espèces connues de fourmis sont représentées par environ $10^{15}$ individus! (BUSH, 1994)

La division pratique entre diversité génétique et diversité spécifique est artificielle. La distinction entre diversité spécifique et diversité écosystémique est également artificielle puisque ce sont les différences de composition et d'abondance relative d'espèces qui différencient les écosystèmes les uns des autres. Le problème réside dans la manière de tenir compte de l'interdépendance des trois niveaux de la biodiversité, ce qui nécessite une approche pluridisciplinaire. La biogéographie, vue sous l'angle des géographes, s'occupe essentiellement de la diversité spécifique et surtout écosystémique aussi, n'évoquerons-nous pas la diversité génétique qui reste le fait des biologistes.

L'expression "diversité biologique" fait souvent penser à la diversité spécifique, c'est-àdire au nombre d'espèces présentes en un lieu. La majorité des recensements dits de biodiversité sont effectivement des relevés d'espèces. Les scientifiques établissent deux listes d'espèces pour un lieu donné, celle des espèces rencontrées et celle des espèces endémiques, qui ne se rencontrent que sur le territoire en question. Cette seconde liste met en évidence l'unicité du lieu étudié (espèces caractéristiques).

\subsection{Le concept d'espèce: un changement de paradigme}

Le concept d'espèce est l'un des sujets problématique en biologie depuis un siècle. Aujourd'hui, deux principaux concepts s'opposent, le concept biologique et le concept phylogénétique. Selon le concept biologique, c'est l'isolement reproductif d'un groupe d'individus qui en fait une espèce. Ce concept dominant fait l'objet de critiques qui vont du problème philosophique que les espèces ainsi définies n'existent pas en soi mais seulement en fonction de l'existence d'autres espèces, jusqu'au problème pratique qu'il est impossible de déterminer si des populations apparentées allopatriques (géographiquement isolées) seraient capables de se reproduire si elles entraient en contact.

Les relations évolutives de plusieurs populations sont représentées sous la forme d'arbres phylogénétiques. Le concept phylogénétique présente l'espèce comme le plus petit groupe irréductible d'organismes différenciable de tout autre groupe de mème nature et dont les membres partagent les mémes liens de filiation (TASSY, 1991). Ce concept assez récent met l'accent non plus sur la reproduction mais sur la différenciation des unités spécifiques et sur la parenté de leurs membres. Toute espèce valide sous le concept biologique sera reconnue sous le concept phylogénétique, son isolement reproductif faisant la preuve de l'existence de caractères taxonomiques. Cependant, nombre de populations apparentées mais n'ayant pas atteint le seuil de l'incompatibilité reproductive seront traitées comme espèces distinctes par les adhérents du concept phylogénétique, si elles sont différenciables l'une de l'autre, alors qu'elles seront vues comme sous-espèces, formes ou variétés suivant le concept biologique. Le concept phylogénétique permettrait une meilleure compréhension des schémas évolutifs et éviterait d'inclure au sein d'une même espèce des populations qui ne sont pas génétiquement les plus proches (NIXON \& WHEELER, 1990). Mais ce concept fait, lui aussi, l'objet de certaines critiques. La plus évidente, c'est que toute population et même tout individu peut être élevé au rang d'espèce. L'adoption du concept phylogénétique aurait un effet marqué sur l'étude de la diversité biologique. Les oiseaux 
comprennent près de 9000 espèces "biologiques" qui correspondent à près de 20000 espèces "phylogénétiques"

\subsection{Le mythe de l'inventaire global}

La diversité spécifique peut être évaluée par l'étude d'une biocénose, d'une région plus ou moins étendue ou par celui d'un groupe systématique. La mesure de la diversité des biocénoses reste délicate car le nombre d'espèces présentes peut atteindre plusieurs dizaines de milliers d'espèces dans des milieux complexes comme la forêt équatoriale (GENTRY, 1988). On ne connait actuellement qu'une infime partie de la biodiversité réelle. Pour ne prendre que le cas des arthropodes habitant la canopée des forêts tropicales, ERWIN (in WILSON et PETER, 1988; ERWIN, 1983) estime leur nombre à une trentaine de millions d'espèces, nombre récemment réduit à 8 millions! Cet auteur a trouvé 163 espèces de coléoptères dans la canopée d'une seule espèce d'arbre. La faune benthique des abysses océaniques. presque entièrement inconnue, pourrait compter des centaines de milliers d'espèces et plusieurs millions d'espèces de bactéries (ANGEL, 1993; GRASSLE, $1991 \mathrm{a}, 1991 \mathrm{~b})$. En outre, les biocénoses n'ont pas de limites spatiales bien marquées. Les groupements effectués à partir d'animaux plus ou moins mobiles ne coïncident presque jamais avec ceux définis à l'aide de la végétation. Plus pratiquement, on utilisera un taxon comme la famille ou l'ordre pour évaluer la diversité. La mesure de la diversité doit aussi tenir compte des effectifs car à nombre égal d'espèces, un peuplement a une diversité plus élevée si toutes les espèces qui le composent ont à peu près les mèmes effectifs. On utilise pour cela un certain nombre d'indices comme celui de Shannon. Le nombre actuel des espèces connues approche 1,8 millions, et certaines estimations de la biodiversité globale avancent le nombre de 100 millions voire nettement plus encore (GROOMBRIDGE, 1992, 1994). Les angiospermes dominent le règne végétal et chez les animaux, les insectes comptent plus de la moitié des espèces connues.

Il faut comprendre que recenser l'ensemble des espèces animales et végétales présentes en un lieu, même de petite taille, est une entreprise insurmontable. Si l'on étudie que des groupes bien connus, tels les oiseaux, les mammifères ou les plantes vasculaires, le travail reste possible. Mais le travail devient trés complexe si l'on inclut des groupes très diversifiés, difficiles à identifier, ou peu connus, ce qui correspond à l'immense majorité des groupes d'organismes vivants. Un très grand investissement en taxonomie et en techniques d'échantillonnage et d'identification serait nécessaire avant qu'on puisse espérer produire un inventaire exhaustif d'un territoire mème de petite taille. Le premier inventaire "global" de la diversité spécifique d'un territoire a été récemment entrepris. Le projet ATBI (All Taxa Biodiversity Inventory) vise à établit le catalogue des espèces présentes sur le territoire du parc naturel de Guanacaste au Costa Rica $\left(110 \mathrm{~km}^{2}\right)$ et coûte près de 100 millions de \$US! (PARIZEAU, 1997).

\subsection{Bio-indicateurs et éco-indicateurs}

La récolte et la détermination de toutes les espèces vivant dans une région est une táche irréalisable et la recherche d'un groupe systématique qui indique l'ampleur de la diversité est un moyen qui s'impose. A cause du manque d'experts pour couvrir de nombreux groupes, les scientifiques utilisent des groupes indicateurs dans l'étude de la biodiversité (BLANDIN, 1986). Les indicateurs optimaux sont des groupes facilement observables et identifiables, dont le catalogue des espèces est relativement bien établi et dont la taxinomie est stable. Pour ces raisons, la plupart des études de la biodiversité marine sont basées sur des relevés des groupes de poissons ou de coraux, celles en biodiversité terrestre sur les groupes 
de plantes vasculaires, d'insectes et d'oiseaux. Paradoxalement, la représentativité de ces groupes n'a fait l'objet de presque aucune analyse. La question est de déterminer dans quelle mesure les résultats obtenus pour les poissons et les algues sont représentatifs de la biodiversité globale et, sinon, quels autres groupes pourraient donner des résultats qui le seraient. Certaines espèces exercent un rôle essentiel tel que leur disparition entrainerait l'extinction en chaines d'autres espèces, déstabiliserait le système et le ferait basculer dans d'autres dynamiques. MILLS (et al. 1993) reconnaissent cinq catégories d'espèces clés:

les prédateurs clés dont l'impact sur leurs proies favorise la coexistence de ces dernières;

les proies clés capables de supporter de fortes pression de prédation. Leur fonction est de soulager les pressions de prédation sur des proies alternatives démographiquement moins fortes;

les mutualistes clés qui assurent des fonctions essentielles de pollinisation et de dispersion de la végétation;

les hôtes clés qui garantissent la survie d'espèces spécialistes;

les modificateurs clés qui entretiennent, par leur action physique et biochimique, l'hétérogénéité de l'environnement.

Hormis la question de déterminer ce qu'est une espèce et quelle est la fiabilité des indicateurs actuels de la biodiversité, les recensements spécifiques sont compliqués par de nombreux aspects pratiques, liés soit aux méthodes soit aux plans d'échantillonnage. Le défi principal semble ètre d'établir des techniques d'analyse qui permettront de combiner des ensembles de données de qualité variable, soit parce que différents sites auront été recensés différemment, ou parce que différents groupes d'animaux ou de plantes auront été recensés plus ou moins régulièrement en un mème lieu.

\subsection{Comment déterminer la bonne échelle spatiale d'analyse?}

Concernant le plan d'échantillonnage, la détermination de l'échelle spatiale de mesure est un problème fondamental. Pour un espace écologiquement homogène, il existe une relation croissante entre surface échantillonnée et nombre d'espèces recensées. Ainsi dans une forèt parvenue à maturité, il est rare de trouver plus d'une espèce d'arbre sur une surface d'un mètre carré. Ce nombre augmente graduellement avec l'accroissement de la surface d'échantillonnage, jusqu'à atteindre un plateau correspondant au nombre total d'espèces présentes dans la forêt (courbe aire-espèces). Il est donc important de déterminer la surface minimale à échantillonner pour obtenir un indice fiable de la diversité du milieu étudié. La façon la plus fréquente de présenter la richesse spécifique d'un lieu est d'indiquer le nombre d'espèces d'un ou de plusieurs groupes taxonomiques qui y est rencontré. Lorsque le lieu considéré est une surface écologiquement homogène, cette valeur est appelée diversité alpha. Les forèts tropicales, par exemple, sont riches en espèces et possèdent une diversité alpha élevée.

\subsection{Le problème de l'hétérogénéité}

Un second aspect est celui de l'hétérogénéité écologique: plus il y a d'habitats différents sur une surface, plus le nombre d'espèces présentes sera grand. Pour tenir compte de ce fait, les écologues utilisent la notion de diversité bêta, qui reflète le taux de changement de composition spécifique entre habitats. Un haut degré de diversité bêta sur un territoire entrainera une grande richesse en espèce mème si chaque habitat n'est pas trés riche. On parle aussi de diversité gamma pour décrire les changements de composition spécifique entre grands territoires, entre continents par exemple. La diversité gamma est la diversité spécifique totale d'une région étendue. 
Parce que plusieurs espèces animales et végétales sont spécialisées, la diversité gamma sera d'autant plus grande que le nombre d'habitats différents dans la région étudiée sera élevé. Il existe une relation entre diversité alpha et gamma qui est la proportion moyenne d'espèces qui diffèrent entre habitats. Ce taux de changement ou de remplacement correspond à la diversité bêta. Il est facile de comprendre qu'une forte diversité gamma peut correspondre à de nombreuses réalités (diversité alpha élevée et bêta faible, diversité alpha et bêta moyenne, ...) et c'est ce que l'utilisation simultanée des indices alpha et bêta permet de comprendre.

\section{Diversité des écosystèmes}

L'approche écosystémique peut laisser penser que la définition de l'écosystème et son identification ne pose pas de problème, ce qui est inexact. Il faudrait que la nature soit organisée du point de vue écologique en entités discrètes et discontinues pour pouvoir la diviser de manière non ambiguë. Mais la nature est en réalité beaucoup plus proche d'un continuum que d'une mosaïque d'unités écologiques bien différenciées. Dans le domaine benthique, par exemple, il est difficile de déterminer où s'arrête l'étage infralittoral et où commence l'étage circalittoral. Malgré tout, il est évident que certaines dichotomies ne posent pas de problèmes: les marécages, les forêts, et les déserts sont trois types d'habitats faciles à distinguer.

\subsection{Les définitions de l'écosystème}

On peut définir l'écosystème comme un "complexe dynamique formé de communautés de plantes, d'animaux et de micro-organismes et de leur environnement non vivant qui, par leur interaction, forment une unité fonctionnelle" Cette définition légale correspond à celles que l'on trouve dans les traités d'écologie classiques
(RAMADE, 1992, 1994). Le problème réside dans la difficultés de définir ce qu'est une communauté. Une définition minimale acceptée des biologistes est qu'il s'agit d'une association d'espèces qui interagissent entre elles, occupent un mème environnement et forment un groupe relativement indépendant d'autres associations semblables. Les biologistes utilisent généralement le terme communauté pour désigner des ensembles d'espèces apparentées du point de vue taxonomique ou au niveau trophique (GRABHERR, 1999). On entendra donc parler, pour le milieu marin, de communautés d'algues brunes ou de poissons, et de communautés de filtreurs ou de brouteurs.

Une part de subjectivité est inévitable dans la détermination de ce qui constitue des écosystèmes différents et pour en établir les limites spatiales. Cette subjectivité est évidente dans le choix à faire entre généralité et précision lors de l'établissement de la liste des écosystèmes. Le terme "forêt", par exemple, a une valeur heuristique très limitée, car il correspond à des réalités écologiques extrêmement différentes, depuis la Floresta Amazónica riche en biodiversité, aux forêts d'Araucaria angustifolia presque monospécifiques, de la Mata Atlântica à la Caatinga et de la Restinga aux mangroves littorales. Ces types de végétation diffèrent presque en tout points: les espèces qui les composent, leurs structures végétatives, les types de sols, et les conditions climatiques et écologiques présentes. Il faudra donc distinguer plusieurs écosystèmes forestiers. Les écosystèmes sont donc définis habituellement sur la base des différences de composition spécifique et des conditions environnementales significatives.

\subsection{Les indices de diversité}

Les scientifiques utilisent l'indice de diversité bêta pour identifier la présence de plus d'un écosystème dans une région, et pour déterminer leurs limites spatiales. La diversité 
bêta indique le changement de composition spécifique dans l'espace. Si la comparaison de deux sites indique une haute diversité bêta, deux écosystèmes sont présents. II n'est souvent pas nécessaire d'obtenir des données détaillées pour localiser la transition entre écosystèmes. Un lac et une forèt sont des écosystèmes si distincts que tous peuvent les identifier et en indiquer les limites. Le recours à des données quantitatives précises et aux techniques statistiques de classification hiérarchique est utile dans le cas où il y aurait chevauchement des écosystèmes, ou pour comparer différents territoires. L'aspect hiérarchique ouvre la porte à une grande précision dans l'étude d'aires géographiques limitées, et à une certaine généralité pour les comparaisons de territoires de plus grande dimension.

\subsection{La cartographie des limites spatiales des écosystèmes}

Etablir la liste et les limites spatiales des écosystèmes d'une région suppose l'existence de données sur la présence, l'absence et l'abondance relative des espèces (Tableau 1). C'est un travail qui demande souvent un temps et des ressources humaines et financières importantes. Depuis peu, les systèmes de télédétection sont utilisés pour les régions difficilement accessibles. Les cartographies obtenues ne sont pas basées sur des données de composition spécifiques, mais sur des indicateurs non-biologiques comme le taux d'absorption et d'émission de des longueurs d'onde. Ces cartographies indirectes doivent donc être vérifiées par des observations directes. L'avènement des Systèmes d'Information Géographique a permis de

Tableau 1: Classification dimensionnelle des systèmes "naturels"

\begin{tabular}{|c|c|c|c|}
\hline Niveaux & Dimensions & Exemple marin & Exemple continental \\
\hline Zone & $10000-2000 \mathrm{~km}$ & $\begin{array}{l}\text { Régions tropicales } \\
\text { océaniques }\end{array}$ & $\begin{array}{l}\text { Régions tropicales } \\
\text { continentales }\end{array}$ \\
\hline Domaine & $2000-1000 \mathrm{~km}$ & $\begin{array}{l}\text { Plateforme continentale } \\
\text { du Brésil }\end{array}$ & $\begin{array}{l}\text { Chaîne bordière } \\
\text { montagneuse du } \\
\text { Brésil }\end{array}$ \\
\hline Province & $1000-100 \mathrm{~km}$ & Littoral Sud de Bahia & Serra do Mar \\
\hline Région & $100-1 \mathrm{~km}$ & Archipel d'Abrolhos & Mata Atlântica \\
\hline Géosystème & $100-1000 \mathrm{~m}$ & Platier corallien & Forêt de versant \\
\hline $\begin{array}{l}\text { Ecosystème } \\
\text { Biogéocénose }\end{array}$ & $10-100 \mathrm{~m}$ & Pàté de corail & Arbre à épiphytes \\
\hline Biocénose/Faciès & $<10 \mathrm{~m}$ & $\begin{array}{l}\text { Biocénose à Mussismilia } \\
\text { braziliensis }\end{array}$ & $\begin{array}{l}\text { Biocénose à } \\
\text { Broméliacée }\end{array}$ \\
\hline Microbiocénose & $<\mathrm{lm}$ & Surface du corail & Feuilles de Bromélia \\
\hline
\end{tabular}


progresser dans ce domaine et les SIG peuvent et doivent servir à intégrer ces deux types de données et les traiter.

\subsection{Une absence de consensus}

La quantification de la biodiversité reposent souvent sur des présupposés non testés (la représentativité des quelques gènes échantillonnés pour l'étude de la variabilité génétique), sur l'acceptation de concepts discutables (le concept d'espèce), ou sur des protocoles non standardisés (les problèmes d'échelles spatiales). Par conséquent, les estimations de la richesse biologique proposées actuellement doivent ètre utilisées et interprétées avec prudence.

Un autre problème vient du fait que l'effort d'échantillonnage de la biodiversité est biaisé en faveur de certains groupes taxinomiques et en faveur de certaines régions géographiques. Les groupes plus faciles à percevoir sont davantage étudiés. Les oiseaux ont été plus étudiés que les vers. Au plan géographique, les régions les mieux connues sont les aires tempérées du monde occidental, là où sont concentrés la plupart des scientifiques et les moyens financiers.

L'étude de la diversité inclut aussi l'étude d'une autre composante fondamentale: celle de l'écodiversité. Il est nécessaire d'étudier les fonctions qu'exercent les espèces dans les écosystèmes comme les cycles biogéochimiques, les processus de structuration des populations, des communautés et des écocomplexes (BLANDIN \& LAMOTTE, 1988). On peut voir l'intérêt d'identifier les espèces qui sont essentielles au fonctionnement de l'écosystème et de détecter les espèces ou groupes d'espèces qui en sont les principaux acteurs.

\section{Biodiversité et biogéographie}

L'ensemble des estimations de nombre d'espèces présentées est basées sur l'acceptation d'un concept d'espèce proche du concept biologique. L'utilisation du concept phylogénétique de l'espèces augmenterait significati- vement le nombre d'espèces de chaque groupe, mais ne changerait pas les grandes tendances. Certains auteurs fournissent bien souvent des chiffres nettement supérieurs: 3000000 espèces de bactéries, 10000000 espèces d'algues. 1000000 espèces de nématodes ou 100000000 espèces d'insectes! (HAMMOND. in GROOMBRIDGE, 1992). On connait actuellement près de 1800000 espèces animales et végétales et il en existerait au minimum 13600000 d'après GROOMBRIDGE (1994).

\subsection{Les phénomènes de gradients biogéographiques}

La diversité spécifique est publiée sous formes de listes locales, régionales ou nationales. $\mathrm{Ce}$ contexte géopolitique est artificiel d'un point de vue scientifique, mais il a l'avantage de situer les résultats dans le contexte où ils seront utilisés, la gestion de l'environnement et des richesses biologiques étant de la responsabilité des Etats. Ces données permettent d'établir les grandes tendances de la distribution géographique de la diversité spécifique. Le résultat principal est que la majorité des groupes de plantes et d'animaux terrestres atteignent leur richesse maximale dans les régions tropicales. Ceci est aussi vrai pour les organismes marins, tout au moins pour les organismes benthiques des hauts-fonds. La diversité de papillons et d'orchidées des forêts équatoriales, et celle des poissons des récifs coralliens illustrent bien cette tendance. Certains groupes, cependant, sont plus diversifiés dans les régions tempérées ou polaires; c'est le cas des algues brunes ou des baleines. Malgré ces exceptions, il est incontestable que les régions tropicales renferment une grande majorité de la richesse spécifique de la planète (STEVENS, 1989). On estime par exemple que les forêts tropicales qui ne couvrent que 6 ou $7 \%$ de la surface du globe contiennent plus de 50\% des espèces existantes, peut-être jusqu'à $90 \%$. L'exemple des coraux permet de mettre en évidence des provinces biogéographiques (IndoPacifique et Caraïbes-Atlantique) du milieu marin. 
La province Indo-Pacifique comprend 75 genres et près de 800 espèces alors que la province Caraïbes-Atlantique seulement 20 genres et 84 espèces; 5 genres sont communs aux deux provinces mais aucune espèce. La zone la plus riche est située dans un triangle de biodiversité maximale entre les Philippines, Bornéo et la Nouvelle-Guinée. Les récifs coralliens sont des écosystèmes équivalents, par leur biodiversité et leur complexité, aux forēts tropicales. Il existe dans les récifs de coraux entre 35000 et 60000 (90000) espèces décrites mais ce chiffre ne représenterait que 8 à $14 \%$ de la richesse spécifique réelle (CULOTTA, 1994; HARMELIN-VIVIEN \& BOURLIERE, 1989).

D'autres tendances ont été observées pour la répartition des organismes terrestres, notamment que, à latitude comparable, les sites en altitude tendent à être moins riches. On note aussi une richesse accrue dans les régions pluvieuses. Cette tendance est pondérée par l'influence du régime des pluies; les sites où la saisonnalité est plus prononcée et la saison sèche plus longue, peu importe l'accumulation totale des précipitations annuelles, sont généralement plus pauvres que les sites moins saisonniers. Au niveau des organismes aquatiques, la salinité est un facteur important. En milieu marin on note une diminution importante de la richesse spécifique dès que la salinité est sous le taux de $35 \%$. A l'opposé, dans les écosystèmes d'eau douce la diversité chute quand il y a augmentation de la salinité au-dessus de $2 \%$.

\subsection{Les hots spots de la biodiversité dans le monde}

La cartographie des points chauds de la diversité biologique revêt une grande importance (PRENDERGAST, 1993). Ces lieux sont identifiés en fonction soit d'une grande richesse spécifique totale, soit de leur richesse en espèces endémiques. La liste de ces sites est un outil fondamental pour la gestion et la protection de la diversité biologique.
Il existe douze pays de mégadiversité: l'Australie, le Brésil, la Chine, la Colombie, I'Equateur, I'Inde, I'Indonésie, Madagascar, la Malaisie, le Mexique, le Pérou et la République démocratique du Congo (ex Zaïre); à la suite de l'analyse de leur richesse en espèces de vertébrés, de papilionidés et de plantes vasculaires. Les données de diversité totale masquent souvent le caractère unique de certains sites. Ainsi, le Pérou et l'Equateur sont des centres de forte diversité de mammifères terrestres, avec 271 et 344 espèces présentes respectivement, mais une majorité de ces espèces (208) se rencontrent dans les deux pays. Par contre, les trois-quarts ( 77 sur 105) des espèces de mammifères de Madagascar ne se rencontrent nulle part ailleurs. Aussi, l'établissement de listes de pays et de sites riches en espèces endémiques est essentiel. L'Australie est le pays le plus riche en mammifères (210) et reptiles (605) endémiques, l'Indonésie en oiseaux (356), le Brésil en amphibiens (293) et la Chine en plantes vasculaires (18000) endémiques. Dans le monde, 18 sites comptent à eux seuls 50000 espèces endémiques de plantes vasculaires, soit $20 \%$ de la diversité mondiale de plantes (MYERS, 1988, 1990); 737 espèces d'amphibiens et 892 de reptiles sont endémiques à ces 18 sites, ce qui correspond à $30 \%$ de la diversité totale de la planète et à $13 \%$ de celle de reptiles.

L'endémisme est un aspect de la biodiversité qu'il est nécessaire de prendre en compte. Une espèce endémique est localisée à une région limitée dont l'aire peut varier de quelques dizaines de $\mathrm{m}^{2}$ à quelques centaines de $\mathrm{km}^{2}$ Le taux d'endémisme est élevé pour les iles et les régions tropicales, de mème que dans les régions de climat méditerranéen et dans les massifs montagneux. La délimitation des régions riches en endémiques présente une grande importance pour l'établissement des zones à protéger (Tableau 2). Les centres actuels d'endémisme correspondraient à des types de milieux sans équivalent actuel qui auraient subi 
Tableau 2: Familles de mammifères par provinces biogéographiques

\begin{tabular}{lcccccc}
\hline Régions & Paléarctique & Néarctique & Afrique & Australie & Orientale & Néotropicale \\
\hline Familles & 29 & 23 & 44 & 11 & 31 & 32 \\
Familles endémiques & 1 & 3 & 16 & 10 & 4 & 15 \\
$\%$ Familles endémiques & 3 & 13 & 36 & 91 & 13 & 47 \\
\hline
\end{tabular}

DAJOZ, 1996.

des perturbations répétées causées par les changements climatiques (PRANCE, 1982). Ceci vient à l'appui des théories sur le rôle des perturbations dans l'établissement de la biodiversité.

Il se dégage de l'ensemble de ces données que nous ne connaissons pas plus de $5-10 \%$ de la richesse spécifique de la planète. Les groupes que nous connaissons mieux servent d'indicateurs de diversité biologique et de sa perte réelle ou potentielle. De nombreux scientifiques cherchent actuellement à déterminer si ces indicateurs peuvent être fiables. Il est évident que la richesse spécifique n'est pas répartie uniformément sur la surface de la planète. La distribution non homogène de la diversité biologique est ainsi démontrée par l'existence de points chauds de diversité.

\subsection{L'érosion croissante de la biodiversité}

Le rythme des extinctions va croissant. Depuis 1600, l'Homme a exterminé 151 espèces de mammifères et d'oiseaux. Jusque 1800 , le nombre moyen d'espèces de mammifères disparus par an était de 0,02. Il est de 0.93 pour la période 1901-1906. Des calculs ont été effectués à partir des données relatives aux forêts tropicales dont la surface s'amenuise de $200000 \mathrm{~km}^{2}$ par na (Tableau 3). Le taux moyen d'extinction y est de 17500 espèces par an (WILSON, 1992). Les surfaces primitives des forèts tropicales étaient de $2204.10^{3} \mathrm{~km}^{2}$. Actuellement, elles ne sont plus que de 292.1 $0^{3}$ $\mathrm{km}^{2}$. L'ensemble de ces régions renferme $13,8 \%$ des espèces endémiques de la flore mondiale (DAJOZ, 1996). Si la déforestation se poursuit au rythme actuel, 15 à $20 \%$ des espèces auront disparu des tropiques humides d'ici une trentaine d'années soit 50000 à 100000 espèces par an (BLONDEL, 1995). Ces zones de diversité élevée ont souvent eu un passé géologique complexe, favorable à une intense spéciation.

Il existe quatre grands mécanismes par lesquels l'homme conduit les espèces à l'extinction. Le premier, c'est la fragmentation et la réduction des habitats. Le second, ce sont les prélèvements excessifs sur certaines espèces. Le troisième mécanisme d'atteinte à la diversité, ce sont les introductions d'espèces étrangères dans les écosystèmes. Enfin, le quatrième mécanisme, ce sont les enchainements d'extinctions. Dans ce cas, on ne touche pas à l'espèce, ni à son habitat mais à un élément biologique de son cycle de vie.

La perte de la biodiversité, spectaculaire dans les régions tropicales, touche en fait l'ensemble de la planète. Ce changement global est suffisamment préoccupant pour qu'on cherche à en évaluer les conséquences sur le fonctionnement des écosystèmes et, à terme, sur l'aptitude de la planète à supporter les sociétés humaines. 
Tableau 3: Les zones critiques (hotspots areas) des forèts tropicales

\begin{tabular}{lrrrrr}
\hline Régions & $\mathbf{1}$ & $\mathbf{2}$ & $\mathbf{3}$ & $\mathbf{4}$ & $\mathbf{5}$ \\
\hline Madagascar & 62 & 10 & 6000 & 4900 & 82 \\
Mata Atlàntica (Brésil) & 1000 & 20 & 10000 & 5000 & 50 \\
Equateur (région occidentale) & 27 & 2,5 & 10000 & 2500 & 25 \\
Colombie (Choco) & 100 & 72 & 10000 & 2500 & 25 \\
Amazonie occidentale montagneuse & 100 & 35 & 20000 & 5000 & 25 \\
Himalaya oriental & 240 & 53 & 9000 & 3500 & 39 \\
Péninsule malaise & 120 & 26 & 8500 & 2400 & 28 \\
Nord-Bornéo & 190 & 64 & 9000 & 3500 & 39 \\
Philippines & 250 & 8 & 8500 & 3700 & 44 \\
Nouvelle-Calédonie & 15 & 1,5 & 1580 & 1400 & 89 \\
\hline
\end{tabular}

DAJOZ, 1996.

1: Surface primitive de forèt primaire en milliers de $\mathrm{km}^{2}$. 2: Surface actuelle. 3: Nombre d'espèces présentes dans les forèts originelles. 4: Nombre d'espèces endèmiques dans les foréts originelles. 5: \% d'espèces endémiques.

\subsection{Diversité écosystémique et frag- mentation des habitats}

Il existe une relation entre la diversité écosystémique d'un territoire et l'étendue moyenne des écosystèmes présents, leur contiguité et leur arrangement spatial. L'existence d'un écosystème étant liée à des conditions abiotiques directes, et la diversité des conditions abiotiques étant principalement le reflet de variations topographiques, la diversité des écosystèmes d'une région est étroitement liẻe à des conditions orographiques.

Dans les milieux marins, on note aussi que les régions tropicales sont généralement plus diversifiées que les régions tempérées et polaires. Cette relation est entre autre liée aux contraintes environnementales plus grandes dans les régions extra-tropicales. Des facteurs biotiques semblent aussi ètre en jeu ici. Aux latitudes tropicales, beaucoup d'espèces sont spécialisées, entretenant des liens étroits avec un petit nombre d'autres espèces, ce qui tisse des communautés très hautement entremêlées. La répartition géographique de ces communautés étant limitées par la répartition de leurs éléments les plus localisés, les écosystèmes qu'elles forment couvriront, en moyenne, des surfaces plus restreintes que les communautés formées d'espèces non spécialisées.

Le thème de la fragmentation des habitats naturels est un sujet d'une grande actualité. Même si leurs conclusions ne sont pas toujours généralisables parce que "l'effet surface" et "l'effet d'isolement" des "iles d'habitats" sont variables selon les groupes, les régions et la proportion d'habitat d'origine perdu, de nombreuses études font état d'une réduction des diversités spécifiques sur ces petits espaces morcelés. La fragmentation des habitats a des conséquences sur les diversités locale (alpha) et régionale (gamma) et sur la structure des peuplements. L'étude diachronique de la fragmentation des habitats peut ètre réalisée par télédétection et par l'intermédiaire des SIG. Les biogéographes peuvent apporter beaucoup aux biologistes et écologues dans ce domaine.

Les variations dans l'espace et le temps de la diversité écosystémique sont encore mal comprises et sont actuellement les sujets de réflexion de nombreux scientifiques. Ces variations ont cependant une importance évidente pour la gestion et la protection de la 
diversité biologique. D'aprés la théorie de la biogéographie insulaire de Mac-Arthur et Wilson, l'établissement d'un archipel de parcs de petite ou de moyenne taille serait à privilégier pour les régions les plus diversifiées, alors que quelques grands parcs pourraient ètre plus profitables dans les régions plus homogènes.

\subsection{Vers une gestion intégrée de la biodiversité}

On étudie pratiquement la diversité biologique en la divisant : diversité génétique, des espèces et des écosystèmes. Mais cette division est artificielle, il conviendrait d'intégrer en une valeur unique les estimations de diversité à ces trois niveaux pour obtenir une appréciation globale de la diversité d'un site ou d'une région.

Un problème fondamental se pose ici, celui de l'unité de mesure, qui doit nécessairement être l'unité utilisée pour quantifier la diversité au plus bas échelon d'organisation: le gène. Il est possible de quantifier, sur la mème échelle et avec des techniques compatibles, la différenciation entre gènes d'une même espèce et celle entre gènes d'espèces différentes. L'intégration des niveaux intra- et interspécifiques de la diversité biologique, bien que complexe mathématiquement, semble donc possible. Par contre, il n'est pas du tout clair qu'on pourra quantifier la diversité écosystémique sur une échelle de divergence génétique. Il est donc difficile d'intégrer les trois niveaux de diversité biologique (NOSS, 1990).

Une mesure unique de diversité biologique serait utile au travail des scientifiques, permettant d'identifier les cibles prioritaires des programmes de conservation et les lieux les plus propices pour l'établissement de zones protégées. Il semble toutefois qu'une évaluation parallèle des indices de diversité génétique, spécifique et écosystémique soit plus profitable. Ainsi les taux de diversité d'espèces et d'écosystèmes sont des facteurs primordiaux pour identifier l'endroit où il est préférable d'établir un parc. Par la suite, la question de la diversité génétique devrait entrer en ligne de compte pour définir l'étendue souhaitable du parc, la taille d'un territoire étant liée au nombre d'individus qu'il peut supporter et donc à la variabilité génétique que l'on peu espérer préserver. La diversité des indices de diversité biologique peut donc être traitée comme une richesse, plutôt qu'une limite. Aussi bien dans un contexte d'étude que de gestion de la biodiversité, chaque indice aura son utilité car chacun reflète un aspect particulier de la diversité. Le défi sera d'utiliser chaque fois l'indice le plus approprié à la question posée.

\section{Conclusion}

La biodiversité est étudiée à trois niveaux. Une large gamme de techniques de quantification et d'analyse statistique sont disponibles et répondent bien aux besoins identifiés. Une des limites principale à l'analyse de la diversité est l'absence de consensus concernant le concept d'espèce. Les biologistes espèrent qu'un seul et mème concept pourra ètre utile pour l'étude des mécanismes de diversification (la spéciation).

Nos connaissances sur la biodiversité restent limitées; peu d'espèces ont été étudiées génétiquement, la plupart des espèces animales et végétales sont encore inconnues et la dynamique des écosystèmes est mal comprise. Pourant, les données disponibles permettent quelques généralisations. En premier lieu, on note l'existence d'une grande variabilité génétique chez la majorité des espèces. L'ampleur de cette variabilité change d'une population à l'autre, d'une espèce à l'autre et d'un groupe à l'autre; mais les espèces peu variables sont l'exception plutôt que la règle, contrairement à ce que l'on avait longtemps cru. La diversité spécifique est inégalement distribuée sur la planète, elle aussi, avec une forte concentration dans les régions tropicales. De même, la 
variabilité des écosystèmes n'est pas homogène au plan géographique; elle est déterminée notamment par les paramètres abiotiques.

Les indices relatifs aux diversité génétique, spécifique et écosystémique constituent une ressource pour l'étude et la gestion de la diversité. Les géographes doivent prendre part à ce grand projet scientifique car ils en ont les moyens. L'apport des outils et des techniques d'analyse spatiale et de cartographie est fondamental pour l'étude de la biodiversité. L'étude et la conservation de la biodiversité ne peut ètre réalisée que globalement et de manière interdisciplinaire. Il s'agit d'un enjeu majeur que sciences de la nature et sciences de l'homme et de la société doivent mener à bien.

\section{Remerciement}

L'auteur tient à remercier Andrea de Castro Panizza pour la traduction du résumé et pour l'élaboration du tableau 1.

\section{Références bibliographiques}

ANGEL, M.V. Biodiversity of the pelagic ocean. Conservation biology. 7. 1993, pp.760-70.

BARBAULT, R. Des baleines, des bactéries et des hommes. Odile Jacob, Paris, 1994.

BLANDIN, P., LAMOTTE, M. Recherche d'une entité écologique correspondant à l'étude des paysages : la notion d'écocomplexe. Bulletin d'Ecologie. 1988.

BLONDEL, J. Biogéographie, approche écologique et évolutive. Masson, Paris, 1995.

BUSH, M.B. Amazonian speciation: a necessary complex model. Journal of Biogeography. 21, 1994, pp.5-17

CHAUVET, M., OLIVIER, L. La biodiversité, enjeu planétaire. Sang de la Terre, Paris, 1993.

CULOTTA, E. Is marine biodiversity at risk? Science. 263, 1994, pp.918-20.

DAJOZ, R. Précis d'écologie. Dunod, Paris, 1996.

DEMANGEOT, J. Les milieux "naturels du globe, Masson, Paris, 1998.

ERWIY, T.L. Tropical forest canopies: the last biotic frontier. Bulletin of Entomologist Society of America. 1983, pp. 14-9.

GENTRY, A.H. Tree species richness of upper Amazonian forests. Proc. Nat. Acad. Sc. 85, 1988, pp.156-9.
GRABHERR, G. Guide des écosystèmes de la terre. Ulmer éd., Paris, 1999.

GRASSLE, J.F. Deep sea benthic biodiversity. Bioscience. 41, 199 la, pp.464-9.

GRASSLE, J.F. Marine biodiversity and ecosystem function. Biology international. 23, 1991b, Pp.1-19.

GROOMBRIDGE, B. Global biodiversity. Status of the Earth Living Ressources. Chapman \& Hall, London, 1992.

GROOMBRIDGE, B. Biodiversity Data Sourcebook. World Conservation Press, Cambridge, 1994.

HARMELIN-VIVIEN, M.L., BOURLIERE, F. Vertebrates in complex tropical systems. Springer-Verlag, Berlin, 1989.

MAC NEELY, J.A. Conserving the world's biological biodiversity. UICN, Morges, 1990.

MAGURRAN, A.E. Ecological diversity and its measurement. Croom Helm, London, 1988.

MILLS, L.S., SOULE, M.E., DOAK, D.F. The keystone-species concept in ecology and conservation. Bioscience. 43, 1993, Pp.219-24.

MYERS. N. Threatened biotas: 'hot spots' in tropical forests. The Environmentalist. 8. 1988, pp. 243-56. 
MYERS, M. The biodiversity challenge : expanded hot spots analysis. The Environmentalist. 10, 1990, pp.187-208.

NIXON, K.C., WHEELER, G.D. An amplification of the phylogenetic species concept. Cladistics. 6, 1990, pp.211-23.

NOSS, R.F. Indicators for monitoring biodiversity: a hierarchical approach. Conservation biology. 4, 1990, pp.335-64.

PARIZEAU, M.H. La biodiversité. Tout conserver ou tout exploiter?. Sciences, éthiques, sociétés. De Boeck Université, Bruxelles, 1997

PRANCE, G. Biological diversification in the Tropics. Columbia University Press, NewYork, 1982.

PRENDERGAST, J.R. Rare species, the coincidence of diversity hotspots and conser- vation strategies. Nature. 365, 1993, pp. 335-7

RAMADE, F. Eléments d'écologie, écologie appliquée. Ediscience international, Paris, 1992.

RAMADE, F. Eléments d'écologie, écologie fondamentale. Ediscience international, Paris, 1994.

STEVENS, G.C. The latitudinal gradient in geographic range: how so many species co-exist in the tropics. Amer. Nat. 133. 1989, p. 240-256.

TASSY, P. L'arbre à remonter le temps. Christian Bourgeois éd., Paris, 1991.

WILSON, E.O., PETER, F.M. Biodiversity. Nat. Acad. Press, Washington, DC, 1988.

WILSON, E.O. The diversity of life. Norton \& Company, New-York, 1992.

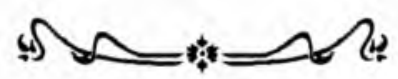

